\title{
Negative Differential Resistance in Doped Armchair Graphene Nanoribbons
}

\author{
Chunmei Liu, Junling Wang, Zhuan Li, Yang Wang, Lili Zhao \\ Department of Fundamental Courses \\ Academy of Armored Force Engineering \\ Beijing 100072, P.R. China
}

\begin{abstract}
Using nonequilibrium Green's functions in combination with density-functional theory, we investigate the electronic transport properties of boron doped armchair graphene nanoribbons. The $I-V$ curve of the device shows an interesting negative differential resistance (NDR) phenomenon. We discover that the NDR is caused by armchair graphene nanoribbons electrodes with boron doped and can be tuned by the length of the AGNR in the central scattering region. This physics finding is helpful for us to design graphene-based nanoelectronic devices.
\end{abstract}

Keywords-boron doping; armchair graphene nanoribbons; electronic transport properties; negative differential resistance

\section{INTRODUCTION}

Graphene nanoribbons (GNRs), are quasi-one-dimensional structures, have attracted a lot of research interests for their unique electronic properties, as well as a promising candidate material for future applications in carbon-based nanoelectronics and molecular devices[1-3]. Based on the different boundary conditions, GNRs are classified into two primary categories: the zigzag graphene nanoribbons (ZGNRs) and the armchair graphene nanoribbons (AGNRs) [4,5]. These GNRs can be either metallic or semiconducting depending on their widths and edge conformations[6]. For instance, ZGNRs are metallic due to the degenerate edge states located on the Fermi level[7]. On the other hand, all AGNRs are semiconductors with different energy gaps[7]. In particular, intensive investigations have been focused on AGNRs, which can be a hopeful candidate material for nanoelectronic devices, such as effect transistors and quantum dots[8].

\section{MODELS AND METHOD}

Here, we report the NDR characteristics of nanodevices fabricated with boron doped AGNR. The width of AGNR is defined by the number of dimer lines and its length by the number of hexatomic rings. We chose the AGNRs as the right and the left electrodes, in which the carbon atoms located at sites labeled with " $\mathrm{B}$ " are substituted by boron atoms. The central scattering region are AGNRs with width $W=7$ and length $L=2,3,4$ as respectively, $L 2, L 3$ and $L 4$ corresponding to different length of AGNRs, L3 system as illustrated in Fig. 1.

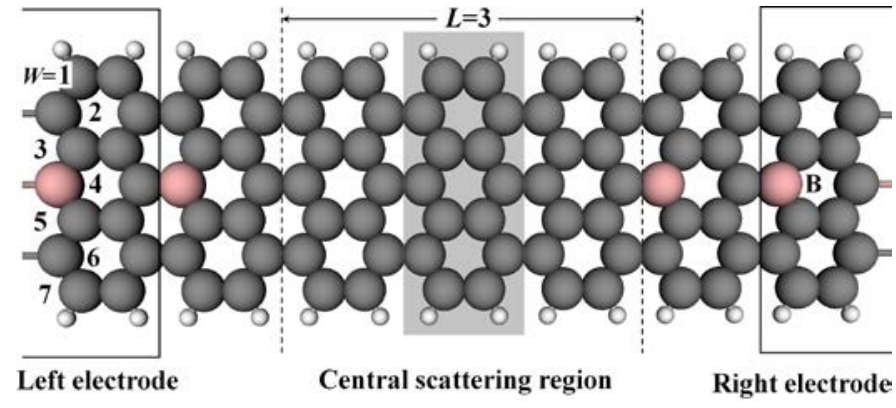

Fig. 1. The two-probe systems of $L 3$. W and $L$ are used to label the width and the length of the AGNR. The left and the right electrodes are boron doped AGNRs.

In our studies, calculations of electronic structure are performed by the ATK package. The exchange-correlation potential is described by the Perdew-Zunger local-density approximation (LDA. PZ). The k-point sampling is 1,1 , and 100 in the $\mathrm{x}, \mathrm{y}, \mathrm{z}$ direction. The transmission spectra through the center region at energy $E$ is expressed as

$$
T(E, V)=\operatorname{Tr}\left[\Gamma_{L}(E, V) G^{r}(E, V) \Gamma_{R} G^{a}(E, V)\right]
$$

where $G^{r}$ and $G^{a}$ are the retarded and advanced Green's functions, $\Gamma_{L}$ and $\Gamma_{R}$ represent the contact broadening functions associated with the left and right electrodes, respectively. The current I can be calculated by LandauerBüttiker formula

$$
I(V)=\frac{2 e}{h} \int_{\mu_{L}}^{\mu_{R}} T(E, V) d E
$$

where $\mu_{\mathrm{L}}$ and $\mu_{\mathrm{R}}$ are the electrochemical potentials of the left and the right electrodes. Under the applied bias $V_{\mathrm{b}}$, the electrochemical potentials of the left and right electrode will be shifted up and down, respectively (namely, $\mu_{R}=\mu-e V / 2$, $\left.\mu_{L}=\mu+e V / 2\right)$. The energy region making contributes to the current integral is $\left[\mu_{L}(V), \mu_{R}(V)\right]$. We refer this region to the bias window or integral window. 


\section{RESULTS AND DISCUSSION}

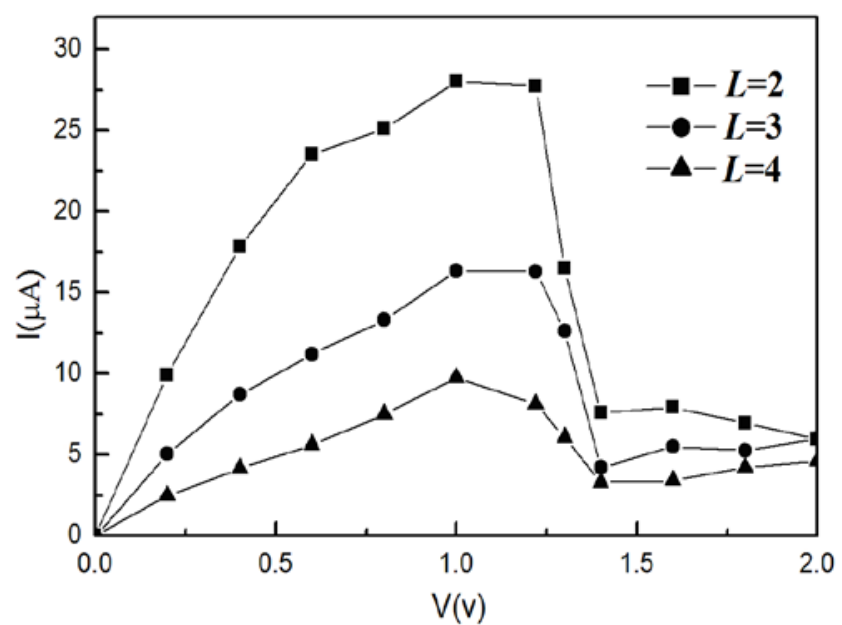

Fig. 2. Calculated currents as a function of the applied bias of L2-L4.

Fig. 2 describes the currents as a function of the applied bias voltages for three systems. We can see that the $I-V$ characteristics of the device are dependent on the length of the AGNR and an NDR peak appears in L2, L3 and L4 systems. Although three systems exemplify robust NDR behavior, the threshold bias voltage is different. In the whole bias voltage range, we can find the currents are $\mathrm{I}_{L 4}<\mathrm{I}_{L 3}<\mathrm{I}_{L 2}$ for the same bias voltage. The device of $L 4$ is the smallest threshold bias voltage, and L2 and L3 system have the same threshold voltage about $1.2 \mathrm{~V}$ on the $I-V$ characteristics, which indicates that the threshold bias voltage can be modulated by the length of the AGNR in the central scattering region.

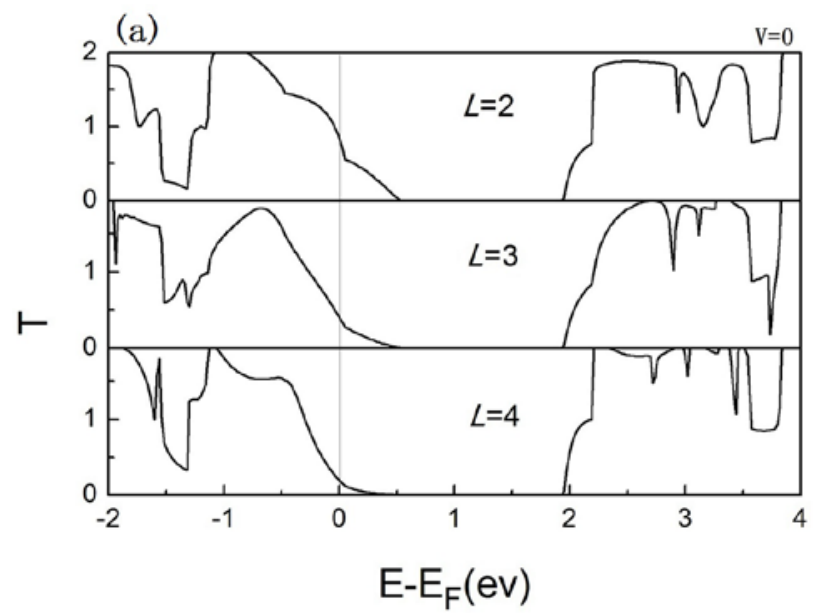

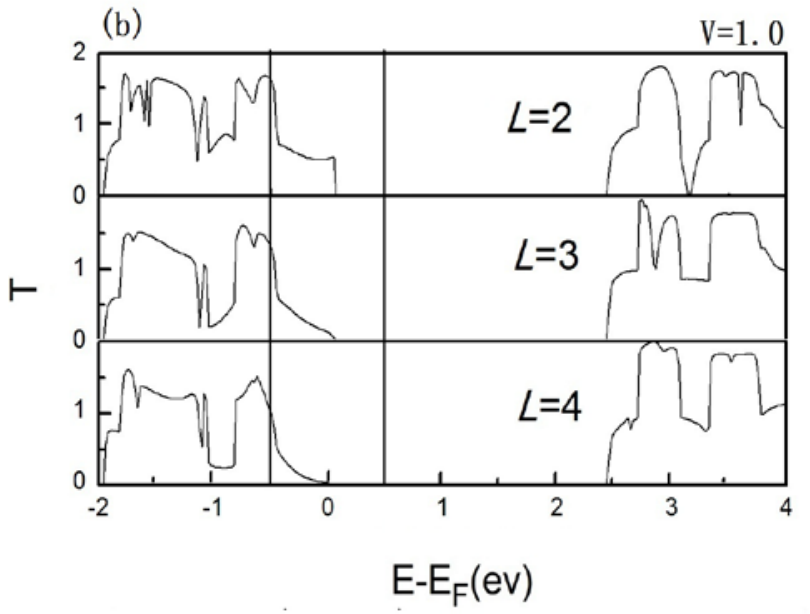

(c)
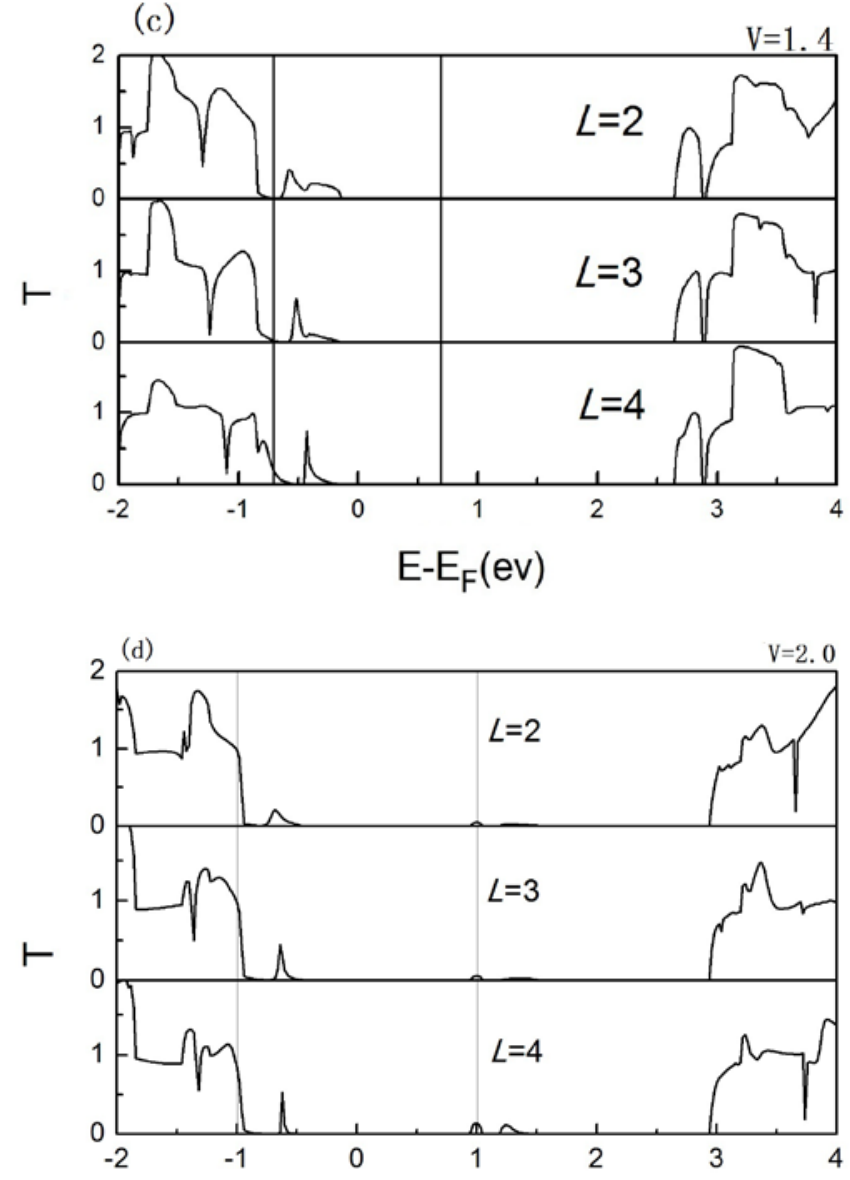

$E-E_{F}(e v)$

Fig. 3. The calculated transmission spectra for all three systems at a bias of $0 \mathrm{~V}$ (a), $1.0 \mathrm{~V}$ (b), $1.4 \mathrm{~V}$ (c) and $2.0 \mathrm{~V}$ (d). The regions between the two vertical solid lines indicate the bias windows. The energy origin is set to be the Fermi level $\mathrm{E}_{\mathrm{F}}$.

To understand the NDR behavior, we calculate the transmission spectrum of the three systems for bias voltage $0 \mathrm{~V}$, $1.0 \mathrm{~V}, 1.4 \mathrm{~V}$ and $2.0 \mathrm{~V}$, respectively shown in Figs. 3. From Landauer- Büttiker formula, we know that only electrons 
within the energy region $\left[\mu_{\mathrm{L}}\left(V_{\mathrm{b}}\right), \mu_{\mathrm{R}}\left(V_{\mathrm{b}}\right)\right]$ contribute to the total current integral. Consider the fact that the $E_{\mathrm{F}}$ is set to be zero, the region of the bias window is actually $[-V / 2, V / 2]$. Thus, the current is determined by $\mathrm{T}(E, V)$ in the bias window. The integral area in the bias window is related to the two factors. One is the transport coefficient and the other is the magnitude of the bias window. It can be seen from Fig. 3 that the integral area inside the bias window of the $L 2$ system is the largest, the $L 4$ system is the minimum, so the currents are $\mathrm{I}_{L 4}<\mathrm{I}_{L 3}<\mathrm{I}_{L 2}$ for the same bias voltage. When the bias voltage increasing from 1.0 to $1.4 \mathrm{~V}$, the bias window at $1.4 \mathrm{~V}$ is much larger than that at $1.0 \mathrm{~V}$, but no additional transmission region will be included into the bias window. Another important point to note is that the transmission coefficients in the new transmission region are getting smaller and smaller. Therefore, the current in voltage range $1.0-1.4 \mathrm{~V}$ decreases rapidly, and the NDR behavior appears in this voltage range. As the bias is further increased to $2.0 \mathrm{~V}$, the new transmission peak enters into the bias window, so the integral area is slightly increased and the current at $V_{b}=2.0 \mathrm{~V}$ is much larger than that at the $V_{b}=1.4 \mathrm{~V}$.

In order to understand the currents behavior clearly, we plot the transmission spectrum and band structures of both left and right electrodes under bias of $0 \mathrm{~V}, 1.0 \mathrm{~V}, 1.4 \mathrm{~V}$ and $2.0 \mathrm{~V}$, as shown in Fig.4(a), (b), (c) and (d). We note that there is a band gap about $2.5 \mathrm{eV}$ for left and right electrodes with the bias of $0 \mathrm{~V}$, shown in Fig.4(a). When the bias is applied to $1.0 \mathrm{~V}$, the energy band for the left electrode moves downward and the right moves upward, shown in Fig.4(b). As the bias is further increased to $1.4 \mathrm{~V}$, the band gap continues to increase, leading to the transmission gap become larger. The current is calculated by the Landauer- Büttiker formula, as in (1), and determined by transmission coefficient in the bias window, resulting in the decrease of the current in voltage range 1.0$1.4 \mathrm{~V}$.

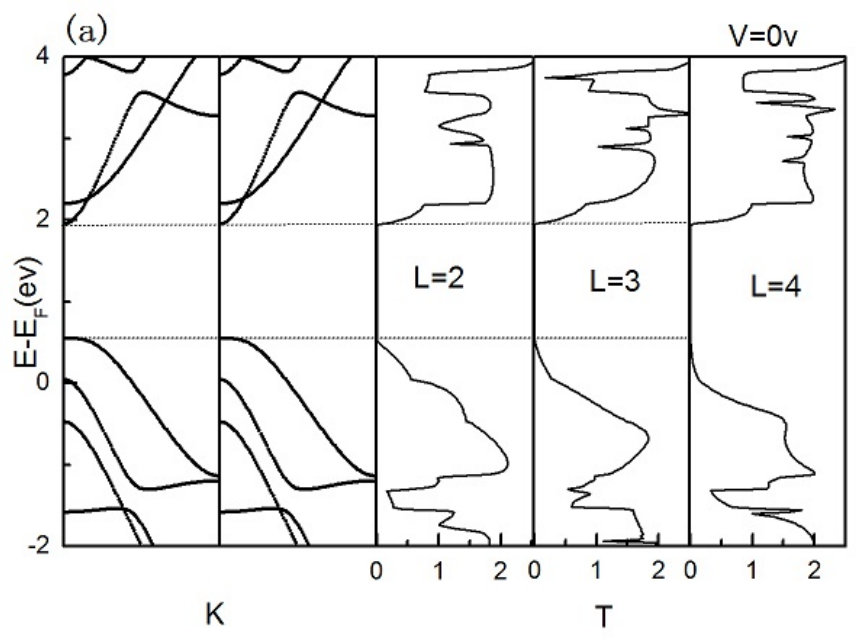

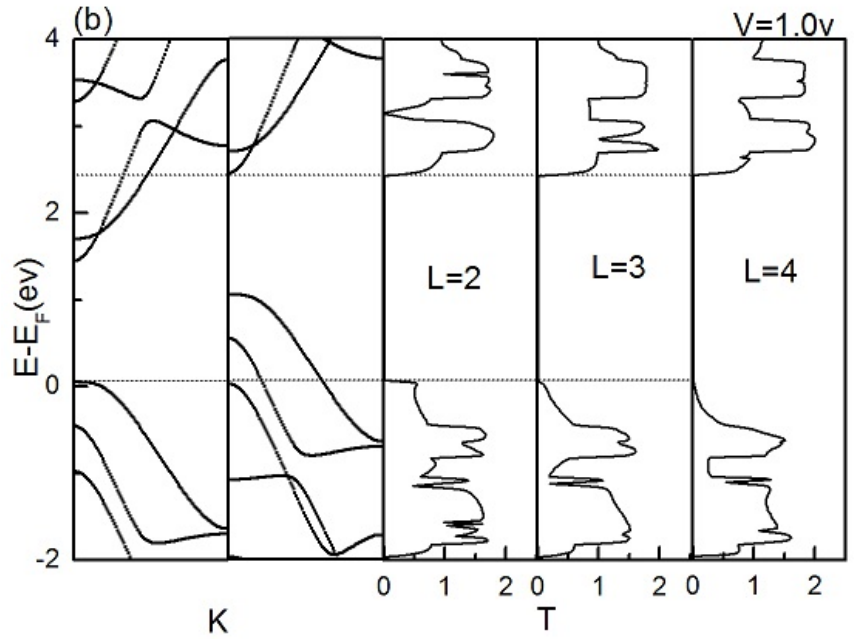
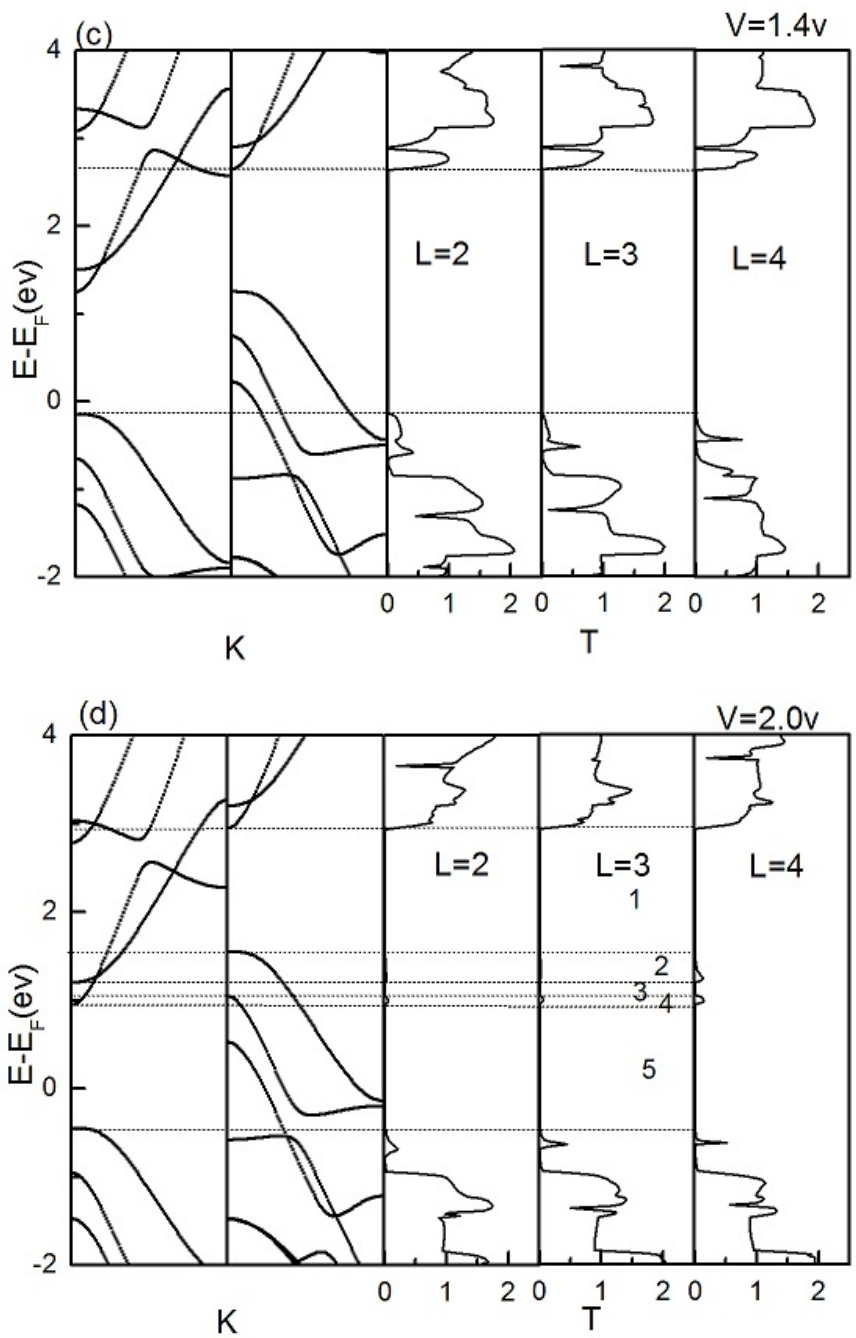

Fig. 4. Band structure for the left electrode (left panel), band structure for the right electrode (middle panel), transmission spectrum (right panel) under bias of $0 \mathrm{~V}(\mathrm{a}), 1.0 \mathrm{~V}$ (b), $1.4 \mathrm{~V}$ and $2.0 \mathrm{~V}$, respectively. 


\section{SUMMARY}

In conclusion, we studied the I-V characteristics and NDR in nano-devices fabricated with boron doped AGNR. Our study shows that NDR behaviors can be observed in such devices and depend on the length of the AGNR in the central scattering region. The NDR can be considered as a result that the transmission coefficients in the transmission region decreases with the increases of the bias window.

\section{REFERENCES}

[1] K.S. Novoselov, A.K. Geim, S.V. Morozov, D. Jiang, Y. Zhang, S.V. Dubonos, I.V. Grigorieva and A.A. Firsov, "Electric field effect in atomically thin carbon films" Science, Vol. 306 , pp. 666-669, 2004.

[2] B.D. Guo, Q. Liu, E.D. Chen, H.W. Zhu, L. Fang and J.R. Gong, "Controllable N-Doping of Graphene" Nano Lett., Vol. 10, pp. 49754980, October 2010
[3] A.K. Geim, K.S. Novoselov: Nat. Mater. Vol. 6, pp. 183-191, June 2007.

[4] Y. Son, M.L. Cohen, S.G. Louie, "Half-metallic graphene nanoribbons" Nature, Vol. 444, pp. 347-349, November 2006.

[5] X. Jia, J. Campos-Delgado, M. Terrones, V. Meuniere, M.S. Dresselhaus, "Graphene edges: a review of their fabrication and characterization" Nanoscale, Vol. 3, pp. 86-95, 2011.

[6] A.H. Castro Neto, F. Guinea, N.M.R. Peres, K.S. Novoselov and A.K. Geim, "The electronic properties of graphene" Rev. Mod. Phys., Vol. 81, pp. 109-162, January-March 2009.

[7] Y. Son, M. L. Cohen, and S. G, "Energy Gaps in Graphene Nanoribbons" Phys. Rev. Lett., Vol 97, pp. 216803-216806, November 2006.

[8] J. Milton Pereira, Jr., P. Vasilopoulos, and F. M. Peeters, “Tunable Quantum Dots in Bilayer Graphene” Nano Lett. Vol. 7, pp. 946-949, March 2007. 